\title{
Replacement of Spectrum Sensing in Cognitive Radio
}

\author{
Zhu Han, Member, IEEE, Rongfei Fan, and Hai Jiang, Member, IEEE
}

\begin{abstract}
Two major challenges exist in the development and deployment of cognitive radio networks: spectrum sensing and hidden terminal problem. In this research, we consider a network structure where the spectrum sensing task is separated from the unlicensed users (secondary users). The service provider for the secondary users needs to place sensing devices within the networks of licensed users (primary users). These sensing devices sense the primary users' activity. The sensing devices also decide whether to admit a secondary user's transmission. A new cognitive cycle is proposed accordingly. The proposed protocol is analyzed using the theory of Lamé curve. The problem of optimally locating sensing devices and the properties of the proposed system are studied for single-user case and multi-user case. For the case without a separate control channel, a lowtemperature handshake technique is proposed for handshakes between the secondary users and the sensing devices. The other advantage of the proposed scheme is from the business model point of view: the expensive sensing devices will be implemented by the cognitive radio service provider, instead of being built in the secondary user devices which are usually consumer products demanding low cost.
\end{abstract}

Index Terms-Cognitive radio, spectrum sensing, hidden terminal.

\section{INTRODUCTION}

$\mathbf{I}$ $\mathrm{N}$ the next generation wireless networks, the frequency spectrum will become one of the most precious resources, and is expected to become congested to accommodate the increasing demand on wireless multimedia communications. To improve the spectrum utilization, cognitive radio [2][3][4] has emerged as a novel approach, since most licensed spectrum has been shown to be severely under-utilized at a particular time and a particular location. In cognitive radio, unlicensed users (termed secondary users or cognitive users) can temporarily utilize the spectrum if the licensed users (termed primary users) are idle.

In cognitive radio networks, a secondary user needs to sense the licensed spectrum to detect any possible activities of primary users. In the literature, spectrum sensing techniques have drawn vast attention in the design and development of cognitive radio networks, and have been extensively investigated [5]-[7]. The objective of spectrum sensing is to detect and further protect possible primary user activities.

Manuscript received May 5, 2008; revised August 6, 2008 and December 15, 2008; accepted March 1, 2009. The associate editor coordinating the review of this letter and approving it for publication was X. Wang.

Z. Han is with the Department of Electrical \& Computer Engineering, University of Houston, Houston, TX 77004, U.S.A. (e-mail: hanzhu22@gmail.com).

R. Fan and H. Jiang are with the Department of Electrical \& Computer Engineering, University of Alberta, Edmonton, Alberta, Canada T6G 2V4 (e-mail: rongfei@ualberta.ca; hai.jiang@ece.ualberta.ca).

The preliminary idea of this work was presented in part [1] at IEEE Wireless Communications \& Networking Conference (WCNC), 2008.

Digital Object Identifier 10.1109/TWC.2009.080603
However, reliable primary user detection and protection is hard to achieve by spectrum sensing [8]. In spectrum sensing, the sensing result of primary transmitter activities is used to predict whether secondary transmission will affect the primary receiver. The physical separation of the primary transmitter and receiver will introduce the hidden terminal problem as follows. When a secondary transmitter is far away from a primary transmitter, but in the vicinity of the primary receiver, it is possible that the secondary transmitter cannot sense the primary transmitter's signal, and decides to transmit its own signal, which will interfere with the primary receiver. This is the notorious hidden terminal problem. Secondary devices with higher detection sensitivity can help in this situation, but with more cost. Cooperative spectrum sensing [9], [10] can alleviate the hidden terminal problem, benefiting from the information exchanges among secondary users. However, a sufficient number of secondary users are needed and distributed so that accurate detection can be achieved.

To overcome the above problems, following the idea of "dedicated sensing network" in [6], we consider a cognitive network structure in which the sensing devices are separated from the secondary users and built by the service provider of the secondary users instead. Each time a secondary user tries to access the spectrum, a handshake takes place between the sensing device and the secondary user. We also propose a lowtemperature mechanism for the handshake between the sensing devices and secondary users in the case when no separate control channel is available. We provide quantitative analysis of the properties of the proposed network. From the business model point of view, the expensive sensing devices will be implemented by the cognitive radio service provider, instead of being built in the secondary user devices which are usually consumer products needing low cost. The major contribution of this research is twofold: important quantitative analysis is given regarding how to optimally deploy the sensing devices so as to guarantee that all primary users can be protected; and effective handshake between the sensing network and the cognitive network is also proposed, with or without a separate control channel.

The rest of this manuscript is organized as follows. The proposed protocol is detailed in Section II, and analyzed in Section III. Numerical results are given in Section IV, followed by discussion for the case without a separate control channel in Section V and conclusion remarks in Section VI.

\section{Proposed Cognitive Protocol}

In cognitive radio, three fundamental cognitive tasks are critical: radio-scene sensing analysis, spectrum analysis, and transmission power control/spectrum management [2]. With the three tasks, the secondary users can acquire knowledge of 


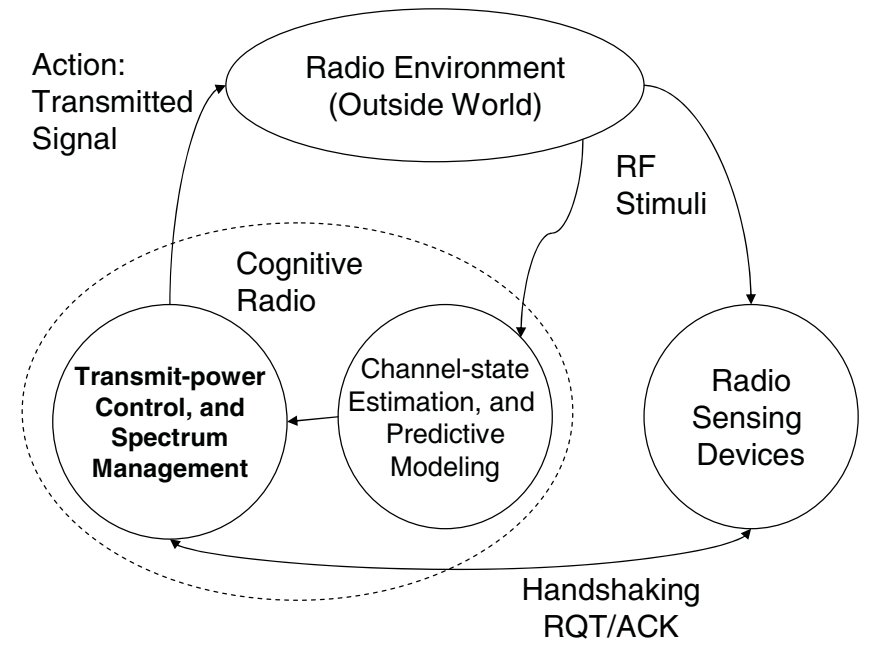

Fig. 1. Proposed cognitive cycle.

the environment and accordingly adapt their spectrum access strategy so as to achieve desired performance and protect the primary users as much as possible.

As aforementioned, the sensing components can be expensive for the secondary users (e.g., when higher detection sensitivity is required so as to alleviate the hidden terminal problem). So we extend the idea of dedicated sensing network that was proposed and briefly described in [6]. Specifically, we consider a cognitive network structure in which the sensing mechanism is not implemented by the secondary users. Instead, the service provider deploys separate sensing devices in the primary network. Those sensing devices are able to detect the primary users' activities and provide admission control to the secondary users for spectrum access. Note that, in [6], a centralized coordinator is adopted to collect sensing results from the sensing devices, and is responsible to provide spectrum availability information to secondary users. On the other hand, in our model, a centralized coordinator does not exist. Rather, each sensing device makes its own decision whether or not admit a nearby secondary user. So our scheme can be deemed distributed.

In Fig. 1, we show the proposed cognitive cycle, which is modified from the traditional cognitive cycle in reference [2]. Compared with the traditional cognitive cycle, within the secondary user, the soft radio part is still maintained. In other words, the cognitive radio devices can still estimate channel state, adjust their transmit power, and manage spectrum access. The difference lies in that the sensing part is moved to the outside sensing devices. It is the sensing devices' role for admission control of secondary users. After admission, a secondary user is responsible to combat the hostile wireless channel and maintain the link quality. If the primary users re-appear after the admission, the sensing devices can also rescind the spectrum admission from the secondary users.

Next we investigate how the scheme can overcome the hidden terminal problem. Note that in wireless local area networks (LANs), the hidden terminal problem can be alleviated by four-way handshake using Request-To-Send/Clear-To-Send (RTS/CTS) [11]. However, it is impossible to implement the
$\mathrm{P} 1$ is transmitting to $\mathrm{P} 2$, and $\mathrm{P} 1$ is hidden terminal to $\mathrm{C}$ traditionally.

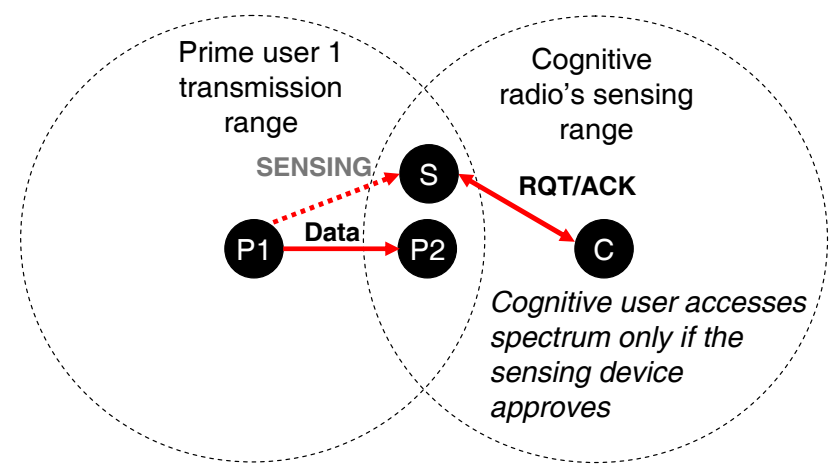

Fig. 2. Solution to the hidden terminal problem by proposed sensing devices.

handshake in cognitive radio, since the primary users will not add additional equipments for handshake. This problem can be solved by the sensing devices in our network model. Consider the network as shown in Fig. 2. Primary user P1 transmits to primary user P2. If the secondary user $\mathrm{C}$ is equipped with sensing ability, it still cannot detect the existence of primary user P1 due to the hidden terminal problem. Instead, a sensing device $\mathrm{S}$ is located in our network model. The frequency band in the licensed spectrum is referred to as the data channel. We assume a separate control channel is available for the handshakes from or to the sensing device. The case without a separate control channel is to be discussed in Section V. Before secondary user C tries to access the data channel, it sends a request (RQT) in the control channel to the nearby sensing device that senses whether any primary user is utilizing the spectrum. If there is any active primary user, the sensing device sends back a veto message in the control channel to the secondary user to deny the spectrum access request. The secondary user will get access to the data channel if it does not detect a veto message in the control channel. By deploying an enough number of sensing devices in the network, the hidden terminal problem is solved and no extra sensing ability is needed for the secondary users.

The proposed system is distributed. The distributed system can be easily implemented since only the request packet needs to be sent from the secondary user and only the veto message needs to be sent from the sensing device to the secondary user. All the message exchanges in the control channel can be based on a collision resolution mechanism such as carriersense multiple access (CSMA). Each sensing device uses its local observation to make a decision whether or not to deny a request. With the presence of primary activities, when multiple sensing devices receive a request from a secondary user, each sensing device will decide to send a veto message in the control channel based on CSMA. A sensing device may cancel its veto message to a secondary user if it detects a veto message from another sensing device that denies the request from the same secondary user.

\section{Performance Analysis}

In this section, a single user case is first investigated, and some properties are discussed. Then, the multi-user case is 


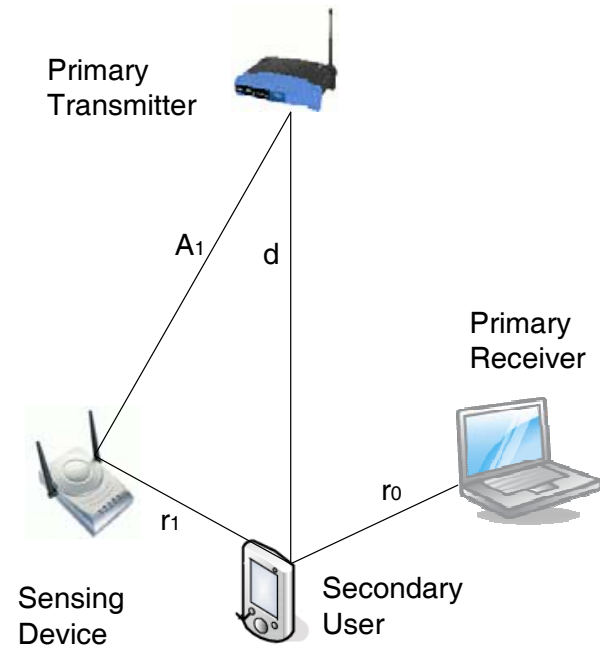

Fig. 3. Analysis model.

studied for topology-aware solution and topology-transparent solution, respectively.

\section{A. Single-User Case}

We first consider the case when there is a secondary user besides a pair of primary transceivers, referred to as the singleuser case. The analysis model is shown in Fig. 3. Suppose the primary transmitter has a distance of $d$ to the secondary user, the sensing device has a distance of $A_{1}$ to the primary transmitter, the secondary user has a distance of $r_{1}$ to the sensing device, and the secondary user has a distance of $r_{0}$ to the primary receiver.

In this research, we assume the propagation loss in each channel (either the data channel or the control channel) consists of path loss with exponent $\alpha$ and Rayleigh fading. Shadowing is not considered ${ }^{1}$.

With the channel model, the probability density function (PDF) of the signal to noise ratio (SNR) (denoted $\gamma$ ) in each channel is given by

$$
f(\gamma)=\frac{N_{0} L^{\alpha}}{P} e^{-\frac{\gamma N_{0} L^{\alpha}}{P}}, \quad \gamma>0
$$

where $P$ is the transmit power, $N_{0}$ is the background noise power, and $L$ is the distance from the sender to the receiver. And the probability that the signal cannot be successfully received is give by

$$
\int_{0}^{\Gamma} f(\gamma) d \gamma=1-e^{-\frac{\Gamma N_{0} L^{\alpha}}{P}}
$$

where $\Gamma$ is a threshold of SNR for successful reception.

From (2), the probabilities for different events are shown as follows.

\footnotetext{
${ }^{1}$ In a traditional cognitive radio network (i.e., when no dedicated sensing devices are adopted), indeed shadowing may result in inaccurate sensing and subsequently cause collisions. However, when the dedicated sensing devices are adopted, shadowing can be addressed as follows. For each primary or secondary transmitter, it is likely that several sensing devices are around. As long as one sensing device can detect the presence of the primary transmitter's activities, the secondary transmitter can be notified (by the sensing device) not to transmit. It is unlikely that severe shadowing exists between all sensing devices and the primary/secondary transmitter.
}

- $\mathrm{P}($ sensing device received $\mid$ primary transmitter sending $)$

$$
P_{S \mid P}\left(A_{1}\right)=\exp \left(-\frac{N_{0} \Gamma_{0}\left(A_{1}\right)^{\alpha}}{P^{p}}\right)
$$

where $P^{p}$ is the power for the primary user, and $\Gamma_{0}$ is the required SNR such that the signal can be detected in the data channel.

- P(sensing device received $\mid$ cognitive user sending RQT in control channel)

$$
P_{S \mid C}\left(r_{1}\right)=\exp \left(-\frac{N_{0} \Gamma_{1}\left(r_{1}\right)^{\alpha}}{P^{h}}\right)
$$

where $P^{h}$ is the power for secondary users for handshake in the control channel, and $\Gamma_{1}$ is the required SNR such that the signal can be detected in the control channel.

- $\mathrm{P}$ (cognitive user received $\mid$ sensing device sending veto in control channel)

$$
P_{C \mid S}\left(r_{1}\right)=\exp \left(-\frac{N_{0} \Gamma_{1}\left(r_{1}\right)^{\alpha}}{P^{s}}\right)
$$

where $P^{s}$ is the power of the sensing device for handshake in the control channel.

- P(primary receiver was interfered with | cognitive user sending in data channel)

$$
P_{P \mid C}\left(r_{0}\right)=\exp \left(-\frac{N_{0} \Gamma_{0}\left(r_{0}\right)^{\alpha}}{P^{c}}\right)
$$

where $P^{c}$ is transmit power of the secondary user in the data channel.

The collision between the primary user and the secondary user happens when there are errors in the sensing or the handshake, if the secondary user's transmission can interfere with the primary receiver. The probability of collision ${ }^{2}$ is then given by

$$
P_{r}^{c}\left(A_{1}, r_{1}, r_{0}\right)=\left[1-P_{S \mid P}\left(A_{1}\right) P_{S \mid C}\left(r_{1}\right) P_{C \mid S}\left(r_{1}\right)\right] P_{P \mid C}\left(r_{0}\right) .
$$

The collision probability is essential for cognitive radio, and should be kept as small as possible, or under a prespecified threshold. Since many parameters are involved in the expression of $P_{r}^{c}\left(A_{1}, r_{1}, r_{0}\right)$, we subsequently investigate four scenarios, each with some parameters fixed: 1) the optimal sensing device location when the locations of the primary transceiver pair are known; 2) the maximal value of $d$ when $r_{0}$ is known; 3) special cases when $r_{1} \rightarrow 0$ or $A_{1} \rightarrow 0$; and 4) feasible location of the sensing device when the locations of the primary transceiver pair and the secondary transmitter are known.

1) Optimal Sensing Device Location: The first problem is to optimally place one sensing device to minimize the collision probability $P_{r}^{c}$. Due to the triangle inequality, the optimal sensing device location should lie on the line between the primary transmitter and the secondary user, i.e., from (3)-(7), we have the optimization problem as

$$
\begin{aligned}
& \arg \min _{A_{1}, r_{1}} P_{r}^{c}\left(A_{1}, r_{1}, r_{0}\right) \\
& \quad=\arg \min _{A_{1}, r_{1}} \frac{\Gamma_{0}\left(A_{1}\right)^{\alpha}}{P^{p}}+\frac{\Gamma_{1}\left(r_{1}\right)^{\alpha}}{P^{h}}+\frac{\Gamma_{1}\left(r_{1}\right)^{\alpha}}{P^{s}} .
\end{aligned}
$$

${ }^{2}$ For simplicity of presentation, "collision" in this research means the collision at the primary receiver due to interference from secondary users. 
Since $A_{1}+r_{1}=d$, we have the optimal location as

$$
A_{1}^{*}=\frac{d}{\left(\frac{\frac{\Gamma_{0}}{P^{p}}}{\left(\frac{\Gamma_{1}}{P^{s}}+\frac{\Gamma_{1}}{P^{h}}\right)}\right)^{\frac{1}{\alpha-1}}+1},
$$

and $r_{1}^{*}=d-A_{1}^{*}$.

2) Maximal Value of $d$ : The second problem is to decide the largest distance from the secondary user to the primary transmitter (i.e., the maximal value of $d$ ) given the distance from the secondary user to the primary receiver (i.e., $r_{0}$ ), under the constraint of a maximal tolerable collision probability (denoted $\varepsilon$ ). From Section III-A1, it is obvious that for a given $\varepsilon$, the maximal value of $d$ is achieved when the sensing device is on the line between the primary transmitter and the secondary user, and its location is given as in equation (9). Any secondary user with a distance to the primary transmitter larger than the maximal value cannot enjoy a collision probability equal to or smaller than the specific one. Substituting equation (7) with corresponding detection probabilities, we have

$$
\begin{aligned}
1- & \varepsilon \exp \left(\frac{N_{0} \Gamma_{0}\left(r_{0}\right)^{\alpha}}{P^{c}}\right) \\
& =\exp \left(-\frac{N_{0} \Gamma_{0}\left(A_{1}\right)^{\alpha}}{P^{p}}-\left(\frac{N_{0} \Gamma_{1}}{P^{h}}+\frac{N_{0} \Gamma_{1}}{P^{s}}\right) r_{1}^{\alpha}\right) .
\end{aligned}
$$

Then according to equation (9) and $A_{1}=d-r_{1}$, the maximal value of $d$ can be expressed as

$$
d_{\max }=\left(-\frac{\ln \left(1-\varepsilon \exp \left(\frac{N_{0} \Gamma_{0}\left(r_{0}\right)^{\alpha}}{P^{c}}\right)\right)}{m \rho^{\alpha}+n(1-\rho)^{\alpha}}\right)^{\frac{1}{\alpha}}
$$

where $m=\frac{N_{0} \Gamma_{0}}{P^{p}}, n=\frac{N_{0} \Gamma_{1}}{P^{s}}$, and

$$
\rho=\frac{1}{\left(\frac{\Gamma_{0}}{\frac{\Gamma}{P P}+\frac{\Gamma_{1}}{P^{h}}}\right)^{\frac{1}{\alpha-1}}+1} .
$$

3) Special Cases: Third, we study the scenario when the sensing device is located right next to the secondary user. So $d=A_{1}$. Because $r_{1} \rightarrow 0$, we have $P_{S \mid C}\left(r_{1}\right) \rightarrow 1$, $P_{C \mid S}\left(r_{1}\right) \rightarrow 1$. Therefore, $P_{r}^{c}\left(A_{1}, r_{1}, r_{0}\right)$ given in (7) is not larger than $\varepsilon$ if the following inequality holds:

$$
P_{S \mid P}\left(A_{1}\right) \geq 1-\varepsilon
$$

since $P_{P \mid C}\left(r_{0}\right) \leq 1$. In other words,

$$
d=A_{1} \leq\left(\frac{-P^{p} \log (1-\varepsilon)}{N_{0} \Gamma_{0}}\right)^{1 / \alpha} .
$$

This means that as long as the distance from the secondary user to the primary transmitter is small enough such that the inequality (14) holds, the service provider can place only one sensing device next to the secondary user so as to prevent the secondary user from interfering with the primary receiver with a collision probability larger than $\varepsilon$.

Next, we study the scenario when the sensing device is located right next to the primary transmitter. So $d=r_{1}$, and $A_{1} \rightarrow 0$. Similarly, $P_{r}^{c}\left(A_{1}, r_{1}, r_{0}\right)$ given in (7) is not larger than $\varepsilon$ if the following inequality holds:

$$
P_{C \mid S}\left(r_{1}\right) P_{S \mid C}\left(r_{1}\right) \geq 1-\varepsilon
$$

which is equivalent to

$$
d=r_{1} \leq\left(\frac{-\log (1-\varepsilon)}{N_{0} \Gamma_{1}\left(\frac{1}{P^{h}}+\frac{1}{P^{s}}\right)}\right)^{1 / \alpha} .
$$

This means that as long as the distance from the secondary user to the primary transmitter is small enough such that the inequality (16) holds, the service provider can place only one sensing device next to the primary transmitter so as to prevent the secondary user from interfering with the primary receiver with a collision probability larger than $\varepsilon$.

4) Feasible Region of the Sensing Device: Finally, we study the feasible region where the sensing device can be placed so that the collision probability is less than the threshold $\varepsilon$, given the locations of the primary transceiver pair and the location of the secondary user. We use the theory of Lamé curve (or superellipse) for analysis. Suppose $P_{r}^{c}\left(A_{1}, r_{1}, r_{0}\right) \leq \varepsilon$, we have

$$
\begin{gathered}
\left\{1-\exp \left[-N_{0}\left(\frac{\Gamma_{0}\left(A_{1}\right)^{\alpha}}{P^{p}}+\left(\frac{\Gamma_{1}}{P^{h}}+\frac{\Gamma_{1}}{P_{s}}\right)\left(r_{1}\right)^{\alpha}\right)\right]\right\} \\
\cdot \exp \left(-\frac{N_{0} \Gamma_{0}\left(r_{0}\right)^{\alpha}}{P^{c}}\right) \leq \varepsilon
\end{gathered}
$$

Here we require $P_{r}^{c}\left(A_{1}^{*}, r_{1}^{*}, r_{0}\right) \leq \varepsilon$.

By some derivation, the feasible region is found to be inside a Lamé curve ${ }^{3}$, which is the geometric figure defined in the Cartesian coordinate system as the set of all points $\left(A_{1}, r_{1}\right)$ with

$$
\left|\frac{A_{1}}{a}\right|^{\alpha}+\left|\frac{r_{1}}{b}\right|^{\alpha}=1
$$

where

$$
\begin{gathered}
a=\left(\frac{c P^{p}}{\Gamma_{0}}\right)^{\frac{1}{\alpha}} \\
b=\left(\frac{c P^{h} P^{s}}{\Gamma_{1}\left(P^{h}+P^{s}\right)}\right)^{\frac{1}{\alpha}},
\end{gathered}
$$

and

$$
c=-\frac{\ln \left[1-\varepsilon \exp \left(\frac{N_{0} \Gamma_{0}\left(r_{0}\right)^{\alpha}}{P^{c}}\right)\right]}{N_{0}} .
$$

In the analysis model, a triangle is formed with the side length of $A_{1}, r_{1}$, and $d$. Suppose the primary transmitter is located at the origin, the sensing device is located at $(x, y)$, and the secondary user is located at $\left(x_{0}, y_{0}\right)$. We have $A_{1}=$ $\sqrt{x^{2}+y^{2}}$ and $r_{1}=\sqrt{\left(x-x_{0}\right)^{2}+\left(y-y_{0}\right)^{2}}$. We have the boundary of the Lamé curve $(x, y)$ as

$$
\left(\frac{\sqrt{x^{2}+y^{2}}}{a}\right)^{\alpha}+\left(\frac{\sqrt{\left(x-x_{0}\right)^{2}+\left(y-y_{0}\right)^{2}}}{b}\right)^{\alpha}=1 \text {. }
$$

\section{B. Multi-User Case}

When there are multiple primary users and multiple secondary users, it is necessary to place multiple sensing devices in the network to guarantee that the collision probability at each primary receiver is lower bounded by a threshold. Generally each combination (primary transmitter, primary receiver,

\footnotetext{
${ }^{3}$ Note that in a Lamé curve, the two axes should be orthogonal. In this research, we still use the term Lamé curve for simplicity, although $A_{1}$ and $r_{1}$ are not orthogonal.
} 
secondary user) has a Lamé curve, in which at least a sensing device should be placed. In the sequel, if a sensing device is placed in a Lamé curve, we say the Lamé curve is covered by the sensing device. Our target is to deploy as few sensing devices as possible to cover all Lamé curves.

It is difficult to obtain a closed-form solution of optimally placing multiple sensing devices, since the placement of new devices will affect the performances of previously deployed devices. In other words, the problem is a complicated assignment problem, which is often NP-hard. Therefore, we aim at suboptimal solutions. In the following, two solutions are proposed, a topology-aware solution and a topologytransparent solution, when the topologies of the primary and secondary networks are known and unknown, respectively.

1) Topology-Aware Solution: The Lamé curve for each combination (primary transmitter, primary receiver, secondary user) is found first. Then the most overlapped area of all the Lamé curves is discovered, and one sensing device is placed in it. After that, the Lamé curves covered by the sensing device are removed. The algorithm continues to place sensing devices until all the Lamé curves are removed.

2) Topology-Transparent Solution: In many scenarios, the exact locations of primary and secondary users are not known a priori. So we propose to divide the area into a number of cells (similar to the cellular systems), and place a sensing device in each cell. The sensing device in each cell is to guarantee that the collision probability of any primary receiver in the cell is lower bounded by a pre-specified threshold $\varepsilon$. In order to determine the size of each cell, we should know the maximum distance (denoted $l$ ) from the sensing device to a primary receiver such that the collision probability of the primary receiver (denoted $P_{r}^{c}(l)$ ) is lower bounded. Assume that a primary receiver is located at the origin $(0,0)$, and the primary transmitter and the secondary user are both uniformly distributed in a circle with the center at the origin and the radius being $R$. Without loss of generality, the sensing device is assumed to be located at $(l, 0)$.

For the collision probability $P_{r}^{c}(l)$, we have

$$
\begin{gathered}
P_{r}^{c}(l)=\frac{1}{\left(\pi R^{2}\right)^{2}} \int_{0}^{2 \pi} \int_{0}^{R} \int_{0}^{2 \pi} \int_{0}^{R} r_{0} r_{2} P_{r}^{c}\left(A_{1}, r_{1}, r_{0}\right) d r_{0} d \theta_{0} d r_{2} d \theta_{2} \\
=\frac{1}{\pi^{2} R^{4}}\left\{2 \pi^{2} R^{2} \int_{0}^{R} r_{0} \exp \left(-\frac{N_{0} \Gamma_{0}\left(r_{0}\right)^{\alpha}}{P^{c}}\right) d r_{0}\right. \\
-\int_{0}^{2 \pi} \int_{0}^{R} r_{2} \exp \left(-\frac{N_{0} \Gamma_{0}\left(r_{2}^{2}-2 r_{2} l \cos \theta_{2}+l^{2}\right)^{\alpha / 2}}{P^{p}}\right) d r_{2} d \theta_{2} \\
\int_{0}^{2 \pi} \int_{0}^{R}\left[r_{0} \exp \left(-N_{0} \Gamma_{1}\left(\frac{1}{P^{h}}+\frac{1}{P^{s}}\right)\left(r_{0}^{2}-2 r_{0} l \cos \theta_{0}+l^{2}\right)^{\alpha / 2}\right)\right. \\
\left.\left.\cdot \exp \left(-\frac{N_{0} \Gamma_{0}\left(r_{0}\right)^{\alpha}}{P^{c}}\right) d r_{0} d \theta_{0}\right]\right\}^{23}
\end{gathered}
$$

where $r_{2}$ and $r_{0}$ represent the distance from the primary transmitter and the secondary user to the primary receiver respectively, and $\theta_{2}, \theta_{0}$ correspond to their arguments. So the design problem becomes:

$$
\max \quad l, \quad \text { s.t. } P_{r}^{c}(l) \leq \varepsilon .
$$

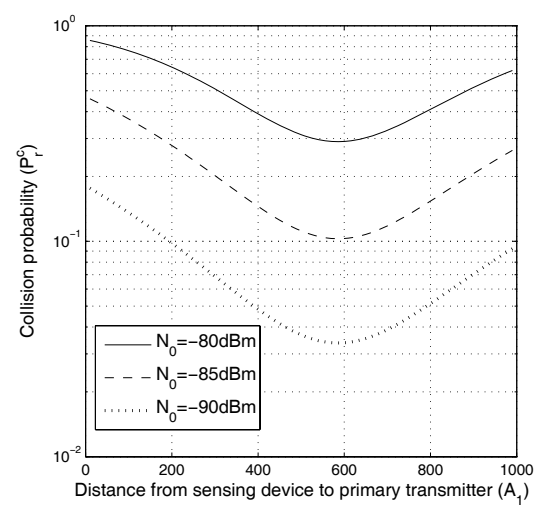

Fig. 4. Collision probability $\left(P_{r}^{c}\right)$ versus distance from the sensing device to the primary transmitter $\left(A_{1}\right)$ (unit: $\mathrm{m}$ ).

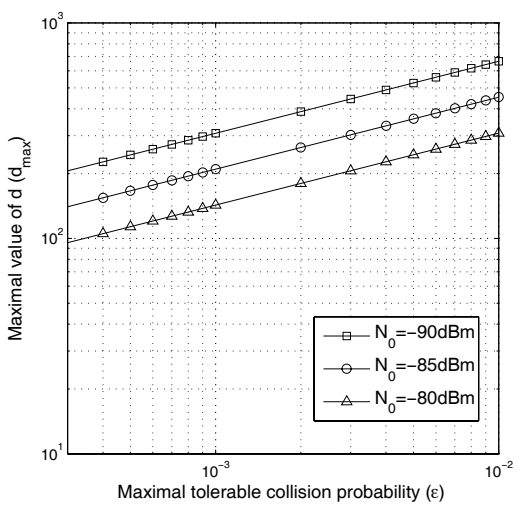

Fig. 5. Maximal value of $d\left(d_{\max }\right)$ (unit: $\left.\mathrm{m}\right)$ versus the maximal tolerable collision probability $(\varepsilon)$.

\section{NUMERicAl RESUlts}

In this section, numerical results are presented to demonstrate the effectiveness of the proposed scheme. The parameters are set up as follows. The primary user power and secondary user power are $P^{p}=P^{c}=P^{s}=P^{h}=0.1$ W. The desired SNRs for the data and control channels are $\Gamma_{0}=\Gamma_{1}=10$. The path loss exponent $\alpha=3$. The thermal noise level $N_{0}$ varies from $-80 \mathrm{dBm}$ to $-90 \mathrm{dBm}$.

Results for the single-user case are first demonstrated. Fig. 4 shows the collision probability $\left(P_{r}^{c}\right)$ versus the distance from the sensing device to the primary transmitter $\left(A_{1}\right)$, when the secondary user is located $1000 \mathrm{~m}$ away from the primary transmitter and $100 \mathrm{~m}$ away from the primary receiver, i.e., $d=1000 \mathrm{~m}$ and $r_{0}=100 \mathrm{~m}$. Here the sensing device is located on the line from the primary transmitter to the secondary user. From Fig. 4, it can be seen that a larger background noise power level $\left(N_{0}\right)$ tends to increase the collision probability. For each $N_{0}$ value, the optimality is achieved approximately at $A_{1}=600 \mathrm{~m}$.

Fig. 5 illustrates the maximal value of the distance from secondary user to primary transmitter (i.e., $d_{\max }$ in (11)) under the maximal tolerable collision probability $\varepsilon$, when $r_{0}=0$ $\mathrm{m}$, i.e., the distance from secondary user to primary receiver is fixed as $0 \mathrm{~m}$, which will promise $d_{\max }$ to be the most 


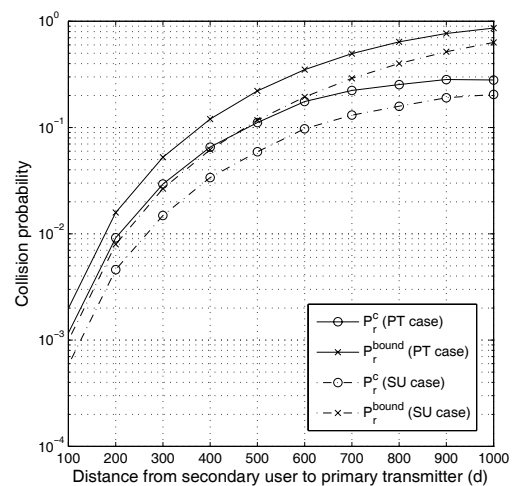

Fig. 6. Collision probability when the sensing device is located right next to the secondary user (SU case) or to the primary transmitter (PT case).

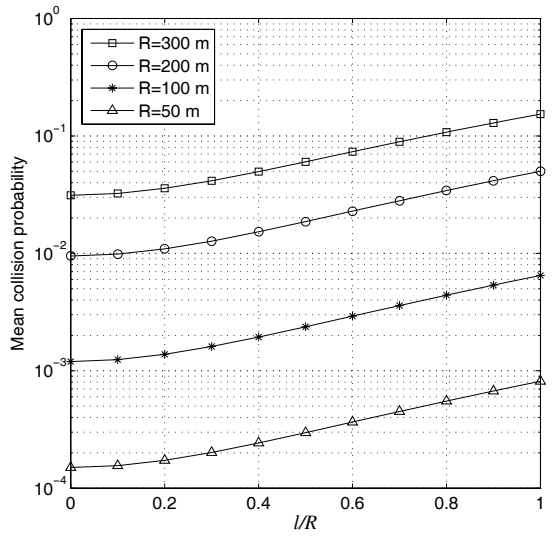

Fig. 7. Mean collision probability $\left(P_{r}^{c}(l)\right)$ versus distance $(l)$ from the primary receiver to the sensing device in the topology-transparent solution.

conservative estimation. It can be seen that a higher $\varepsilon$ allows a larger $d_{\max }$. In other words, with a higher $\varepsilon$, the sensing device can cover a larger area. And a larger background noise power level $\left(N_{0}\right)$ tends to decrease $d_{\max }$.

In the following simulation, the background noise power level $N_{0}=-80 \mathrm{dBm}$. Fig. 6 shows the case when the sensing device is located right next to the secondary user or the primary transmitter. In either case, the primary receiver is uniformly distributed in a $2000 \mathrm{~m} \times 2000 \mathrm{~m}$ square with center at the secondary user. For different distance from the secondary user to the primary transmitter (i.e., $d$ ), we plot the mean value of collision probability $P_{r}^{c}$ (obtained from (7)) and the mean value of the collision probability bound $P_{r}^{\text {bound }}=\varepsilon$ obtained from (14) when the sensing device is right next to the secondary user (represented by "SU case" in Fig. 6) or obtained from (16) when the sensing device is right next to the primary transmitter (represented by "PT case" in Fig. 6). The collision probability increases as the value of $d$ increases.

Further, we demonstrate results for the multi-user case. For the topology-aware solution, we consider the case with one primary transmitter, three primary receivers, and five secondary users. The primary transmitter is located at the origin, while other nodes are uniformly distributed in a circle with the center at the primary transmitter and the radius being
$300 \mathrm{~m}$. It is found that, two sensing devices are needed when the maximal tolerable collision probability $\varepsilon$ is between $10^{-4}$ and $2 \cdot 10^{-4}$, while only one sensing device is necessary when $\varepsilon$ is between $3 \cdot 10^{-4}$ and $10^{-3}$.

Finally, we consider the topology-transparent solution. A primary receiver is at the origin $(0,0)$, and the primary transmitter and the secondary user are both uniformly distributed in a circle with the center at the origin and the radius being $R$. Fig. 7 illustrates the mean collision probability $P_{r}^{c}(l)$ as a function of $l$ with different $R$ values. For each case with an $R$ value, $l$ varies from 0 to $R$. It can be seen that a larger $R$ or a larger $l$ leads to a higher collision probability. Based on Fig. 7, the maximum value of $l$ can be obtained, given the maximal tolerable collision probability $\varepsilon$.

\section{Handshakes without a Separate Control CHANNEL}

In preceding sections, it is assumed that a control channel is available for the handshake between the sensing devices and the secondary users. However, for a large secondary network with potential multiple data channels and a large number of secondary users, it is possible that the control channel may get congested, and thus become the bottleneck. In addition, the separate control channel may not be available. Therefore, in this section, we consider the scenario that the handshake between the secondary users and sensing devices is also performed in the data channel (i.e., no separate control channel is needed). Then a problem arises: how to guarantee that the handshake does not affect primary reception in the data channel. Here we propose a low-temperature handshake to address the problem.

The low-temperature handshake is originally proposed in [12] for a CDMA-based distributed network. Specifically, before a potential transmitter transmits its packet, it should make sure that its transmission will not generate untolerable interference to existing links. So the potential transmitter first sends a probe with a very low power level and a very large spreading gain. The low power level of the probe is to protect existing links, while the large spreading gain is to guarantee that the probe can be detected by existing receivers. After successful reception of a probe, an existing receiver can determine whether the transmitter of the probe will generate untolerable interference, based on the received power level of the probe. If the potential interference is untolerable, the existing receiver will notify the potential transmitter not to transmit.

A similar concept can be adopted in our cognitive radio network model, for the handshake between the secondary users and the sensing devices. The handshake consists of two portions: a request from the secondary user and a potential veto from the sensing device, both at the data channel with very low power level.

If a secondary user has traffic to send, it first sends a request at the data channel. The request has a low transmit power level (much lower than power for its normal data transmission) and is spread by a common request code which has a very large spreading gain and is known in advance to the sensing devices and the secondary network. No bit-information is carried by 
the request. The low power of the request is to guarantee that the tolerable interference temperatures of primary users are not exceeded by the extra interference from the request signal. The spreading gain of the request is large enough such that the sensing devices are able to detect the request.

Each sensing device continuously detects the received power (from primary users) at the data channel. It also scans the common request code for the low-temperature request. If a sensing device detects activities of primary users and also detects a transmission with the common request code, it sends a veto that also has a low transmit power level and is spread by a common veto code, to notify the secondary user of the rejection of its transmission request. No bit-information is carried by the veto. And the spreading gain of the veto is large enough. Therefore, similar to the case with the request, the veto will not generate untolerable interference to primary reception due to the low power, and it can also be detected by the secondary user due to the large spreading gain.

After the secondary user sends the low-temperature request, it scans the common veto code. If no veto is detected, the secondary user is allowed to transmit at the data channel.

Note that the preceding handshake procedure is performed in a distributed manner. Each sensing device uses its local observation to make a decision whether or not to send a veto message. With the presence of primary activities, if multiple sensing devices detect the request from a secondary user, each of the sensing devices will send a veto message using the common veto code. Since no bit-information is carried by the veto messages, the secondary user will detect only one virtual veto message because the energy from all the veto messages will be combined through a Rake receiver. Therefore, the handshake procedure also works well when the request from a secondary user is detected by multiple sensing devices.

We still use the analysis model in Section III-A to study the performance of the low-temperature handshake. We still use $P^{h}, P^{s}$, and $\Gamma_{1}$ to denote the transmit power of the request, the transmit power of the veto, and required SNR for a successful handshake between the sensing device and the secondary user, respectively.

The sensing device keeps sensing the primary activities all the time. Before the secondary user sends the request, the sensing device knows whether or not primary activities exist. So the probability $P_{S \mid P}\left(A_{1}\right)$ keeps the same as in (3), which is a descending function of $A_{1}$. On the other hand, when the sensing device is receiving the request, the primary signal serves as interference with the pdf as $\frac{1}{P^{p} A_{1}^{-\alpha}} \exp \left(-\frac{x}{P^{p} A_{1}^{-\alpha}}\right)$. The pdf of the received request power at the sensing device is $\frac{1}{P^{h} r_{1}^{-\alpha}} \exp \left(-\frac{x}{P^{h} r_{1}^{-\alpha}}\right)$. Let $G_{r}$ and $G_{v}$ denote the spreading gain of the request code and the veto code, respectively. We have

$$
\begin{aligned}
& P_{S \mid C}\left(r_{1}, A_{1}\right) \\
= & \operatorname{Prob}\left\{\text { SNR of the received request } \times G_{r} \geq \Gamma_{1}\right\} \\
= & \int_{0}^{\infty} \frac{1}{P^{p} A_{1}^{-\alpha}} \exp \left(-\frac{x}{P^{p} A_{1}^{-\alpha}}\right) \\
& \cdot\left\{\int_{\left(N_{0}+x\right) \Gamma_{1} / G_{r}}^{\infty} \frac{1}{P^{h} r_{1}^{-\alpha}} \exp \left(-\frac{y}{P^{h} r_{1}^{-\alpha}}\right) d y\right\} d x
\end{aligned}
$$

which is a descending function with respect to $r_{1}$, and an ascending function with respect to $A_{1}$. Similarly we have

$$
\begin{aligned}
& P_{C \mid S}\left(r_{1}, d\right)=\int_{0}^{\infty} \frac{1}{P^{p} d^{-\alpha}} \exp \left(-\frac{x}{P^{p} d^{-\alpha}}\right) \\
& \cdot\left\{\int_{\left(N_{0}+x\right) \Gamma_{1} / G_{v}}^{\infty} \frac{1}{P^{s} r_{1}^{-\alpha}} \exp \left(-\frac{y}{P^{s} r_{1}^{-\alpha}}\right) d y\right\} d x
\end{aligned}
$$

which is a descending function with respect to $r_{1}$, and an ascending function with respect to $d . P_{P \mid C}\left(r_{0}\right)$ keeps the same as in (6).

Lemma 1: The optimal sensing device location should lie on the line between the primary transmitter and the secondary user.

The proof of Lemma 1 is given in the Appendix. Other performance of the low-temperature handshake can be analyzed similar to Section III, and thus is omitted due to the space limitation.

\section{CONCLUSIONS}

Cognitive radio has the potential to improve spectrum efficiency in future wireless multimedia communications. In this research, we consider a network model that replaces expensive spectrum sensing devices in secondary users and avoids the hidden terminal problem. The service provider places the sensing devices in the primary network. The sensing devices detect the primary transmitters' activities and provide admission control for the secondary users. We analyze the problem of collision probability and the sensing device location(s) in single-user and multi-user cases. For the case without a separate control channel, a low-temperature handshake technique is proposed for handshakes between the secondary users and the sensing devices. From the simulation results, we demonstrate the effectiveness of the proposed scheme. This research also provides some helpful insights into the development of cognitive radio networks with low-cost secondary terminals.

\section{APPENDIX: PROOF OF LEMMA 1}

Proof: Assume the primary transmitter and the secondary user are located at the origin and $(0,-d)$, respectively. The optimal sensing device location is at $(x, y)$. So $A_{1}=\sqrt{x^{2}+y^{2}}$, and $r_{1}=\sqrt{x^{2}+(y+d)^{2}}$. The proof consists of three steps.

Step 1 (to prove $y \leq 0$ ): We use proof by contradiction. Suppose $y>0$. We consider an alternative case when the sensing device is located at $(x,-y)$. So for the alternative case $A_{1}^{\prime}=\sqrt{x^{2}+y^{2}}=A_{1}$, and $r_{1}^{\prime}=\sqrt{x^{2}+(y-d)^{2}}<r_{1}$. Thus $P_{S \mid C}\left(r_{1}^{\prime}, A_{1}^{\prime}\right)>P_{S \mid C}\left(r_{1}, A_{1}\right)$, and $P_{C \mid S}\left(r_{1}^{\prime}, d\right)>$ $P_{C \mid S}\left(r_{1}, d\right)$. From (7), it can be seen that the alternative case with sensing device at $(x,-y)$ has a lower collision probability than the original case with sensing device at $(x, y)$. This contradicts the assumption that the optimal sensing device location is at $(x, y)$.

Step 2 (to prove $x=0$ ): We use proof by contradiction. Suppose $x \neq 0$. We consider an alternative case when the sensing device is located at $\left(0,-\sqrt{x^{2}+y^{2}}\right)$. So for the alternative case $A_{1}^{\prime}=\sqrt{x^{2}+y^{2}}=A_{1}$, and $r_{1}^{\prime}=\left|\sqrt{x^{2}+y^{2}}-d\right|$. According to the triangle inequality, $r_{1}^{\prime}<r_{1}$. Then similar to the proof in Step 1, the alternative case has a lower collision 
probability. This contradicts the assumption that the optimal sensing device location is at $(x, y)$.

Step 3 (to prove $y \geq-d$ ): We still use proof by contradiction. Based on Steps 1 and 2, the optimal sensing device location is at $\left(0,-A_{1}\right)$. Suppose $-A_{1}<-d$, i.e., $A_{1}>d$. Then $r_{1}=A_{1}-d$. We consider an alternative case when the sensing device is located at $\left(0,-\beta A_{1}\right)$ where $\beta=\frac{d}{A_{1}+r_{1}}<1$, i.e., $A_{1}^{\prime}=\beta A_{1}(<d)$. Then $r_{1}^{\prime}=d-A_{1}^{\prime}=\beta r_{1}$. So we have

$$
\begin{aligned}
& P_{S \mid C}\left(r_{1}^{\prime}, A_{1}^{\prime}\right) \\
& =\int_{0}^{\infty} \frac{1}{P^{p}\left(\beta A_{1}\right)^{-\alpha}} \exp \left(-\frac{x}{P^{p}\left(\beta A_{1}\right)^{-\alpha}}\right) \\
& \left\{\int_{\left(N_{0}+x\right) \Gamma_{1} / G_{r}}^{\infty} \frac{1}{P^{h}\left(\beta r_{1}\right)^{-\alpha}} \exp \left(\frac{-y}{P^{h}\left(\beta r_{1}\right)^{-\alpha}}\right) d y\right\} d x \\
& =\int_{0}^{\infty} \frac{1}{P^{p}\left(\beta A_{1}\right)^{-\alpha}} \exp \left(-\frac{x}{P^{p}\left(\beta A_{1}\right)^{-\alpha}}\right) \\
& \left\{\int_{\left(N_{0}+x\right) \Gamma_{1} / G_{r}}^{\infty} \frac{1}{P^{h} r_{1}^{-\alpha}} \exp \left(-\frac{\beta^{\alpha} y}{P^{h} r_{1}^{-\alpha}}\right) d \beta^{\alpha} y\right\} d x \\
& \left(\beta^{\alpha} \underline{y \rightarrow}=\int_{0}^{\infty} \frac{1}{P^{p}\left(\beta A_{1}\right)^{-\alpha}} \exp \left(-\frac{x}{P^{p}\left(\beta A_{1}\right)^{-\alpha}}\right)\right. \\
& \left\{\int_{\beta^{\alpha}\left(N_{0}+x\right) \Gamma_{1} / G_{r}}^{\infty} \frac{1}{P^{h} r_{1}^{-\alpha}} \exp \left(-\frac{y}{P^{h} r_{1}^{-\alpha}}\right) d y\right\} d x \\
& =\int_{0}^{\infty} \frac{1}{P^{p} A_{1}^{-\alpha}} \exp \left(-\frac{\beta^{\alpha} x}{P^{p} A_{1}^{-\alpha}}\right) \\
& \left\{\int_{\left(\beta^{\alpha} N_{0}+\beta^{\alpha} x\right) \Gamma_{1} / G_{r}}^{\infty} \frac{1}{P^{h} r_{1}^{-\alpha}} \exp \left(\frac{-y}{P^{h} r_{1}^{-\alpha}}\right) d y\right\} d \beta^{\alpha} x
\end{aligned}
$$

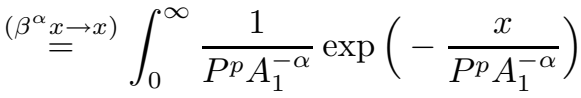

$$
\begin{aligned}
& \left\{\int_{\left(\beta^{\alpha} N_{0}+x\right) \Gamma_{1} / G_{r}}^{\infty} \frac{1}{P^{h} r_{1}^{-\alpha}} \exp \left(-\frac{y}{P^{h} r_{1}^{-\alpha}}\right) d y\right\} d x \\
& \stackrel{\left(\beta^{\alpha} N_{0}<N_{0}\right)}{>} \int_{0}^{\infty} \frac{1}{P^{p} A_{1}^{-\alpha}} \exp \left(-\frac{x}{P^{p} A_{1}^{-\alpha}}\right) \\
& \left\{\int_{\left(N_{0}+x\right) \Gamma_{1} / G_{r}}^{\infty} \frac{1}{P^{h} r_{1}^{-\alpha}} \exp \left(-\frac{y}{P^{h} r_{1}^{-\alpha}}\right) d y\right\} d x \\
& =P_{S \mid C}\left(r_{1}, A_{1}\right) \text {. }
\end{aligned}
$$

Since $A_{1}^{\prime}=\beta A_{1}<A_{1}$, we have

$$
P_{S \mid P}\left(A_{1}^{\prime}\right)>P_{S \mid P}\left(A_{1}\right) \text {. }
$$

Since $r_{1}^{\prime}=\beta r_{1}<r_{1}$, we have

$$
P_{C \mid S}\left(r_{1}^{\prime}, d\right)>P_{C \mid S}\left(r_{1}, d\right) .
$$

From (7) and (26)-(28), it can be seen that the alternative case with sensing device at $\left(0,-\beta A_{1}\right)$ has a lower collision probability than the original case with sensing device at $\left(0,-A_{1}\right)$. This contradicts the fact that the optimal sensing device location is at $\left(0,-A_{1}\right)$.

This completes the proof.

\section{REFERENCES}

[1] Z. Han and H. Jiang, "Replacement of spectrum sensing and avoidance of hidden terminal for cognitive radio," in Proc. IEEE WCNC'08, Las Vegas, NV, USA, Mar.-Apr. 2008, pp. 1448-1452.

[2] S. Haykin, "Cognitive radio: brain-empowered wireless communications," IEEE J. Select. Areas Commun., vol. 23, no. 2, pp. 201-220, Feb. 2005.

[3] E. Hossain, D. Niyato, and Z. Han, Dynamic Spectrum Access in Cognitive Radio Networks. Cambridge University Press, UK, 2009.

[4] H. Jiang, L. Lai, R. Fan, and H. V. Poor, "Optimal selection of channel sensing order in cognitive radio," IEEE Trans. Wireless Commun., vol. 8, no. 1, pp. 297-307, Jan. 2009.

[5] D. Cabric, S. M. Mishra, and R. W. Brodersen, "Implementation issues in spectrum sensing for cognitive radios," in Proc. 38th Asilomar Conf. Signals, Systems Computers, Pacific Grove, CA, Nov. 2004, pp. 772776 .

[6] S. Shankar N, C. Cordeiro, and K. Challapali, "Spectrum agile radios: utilization and sensing architectures," in Proc. DySPAN'05, Baltimore, MD, USA, Nov. 2005, pp. 160-169.

[7] H. Kim and K. G. Shin, "Efficient discovery of spectrum opportunities with MAC-layer sensing in cognitive radio networks," IEEE Trans. Mobile Computing, vol. 7, no. 5, pp. 533-545, May 2008.

[8] S. M. Mishra, A. Sahai, and R. W. Brodersen, "Cooperative sensing among cognitive radios," in Proc. ICC'06, Istanbul, Turkey, June 2006, pp. 1658-1663.

[9] G. Ganesan and Y. Li, "Cooperative spectrum sensing in cognitive radio, part I: two user networks," IEEE Trans. Wireless Commun., vol. 6, no. 6, pp. 2204-2213, June 2007.

[10] K. B. Letaief and W. Zhang, "Cooperative spectrum sensing," invited book chapter in Cognitive Wireless Commun. Networks, Springer, Nov. 2007.

[11] Z. Han and K. J. R. Liu, Resource Allocation for Wireless Networks: Basics, Techniques, and Applications. Cambridge, UK: Cambridge University Press, 2008.

[12] H. Jiang, P. Wang, W. Zhuang, and X. Shen, "An interference aware distributed resource management scheme for CDMA-based wireless mesh backbone," IEEE Trans. Wireless Commun., vol. 6, no. 12, pp. 4558-4567, Dec. 2007 